TG but are lumped together, which creates both a socio-political and behavioural risk issue. Thus, it is important to look at how TG women themselves define and understand the concept of TG in order to provide a context in developing TG-specific health services and HIV prevention programmes.

The methods used were facilitating a self-administered questionnaire to forty-six (46) self-identified TG women, and conducted four (4) focus group discussions to TG women members from community-based organisations (CBOs) in Metro Manila, Cebu City and Davao City.

The findings revealed that majority of the respondents/participants, being affiliated with a $\mathrm{CBO}$, defines TG as persons whose gender identity and/or expression does not conform with their sex assigned at birth. Their differentiation of a TG woman from a transsexual (TS) is that the latter is related more to the concept of body modifications (i.e. hormone replacement therapy, collagen injection and implants). Thus, TG-specific health services should include both empowerment of their TG identities and addressing risky behaviours such as "versatile" sexual role and engaging in various forms of body modifications, especially those who self-inject hormones and collagens. Some TG CBOs coined "transpinay", "transwomen" and "binabae" as a local term for TG women which are useful to reach the unaware Filipino TG women community. Lastly, in order to identify and target TG women clients in peer education, qualifier questions or criteria can be used but always give the target clients the opportunity to self-identify for self-empowerment - both strategies should complement each other.

\section{P3.440 HIV RAPID TESTING IN THE FRAMEWORK OF A STI PREVENTION PROJECT FOR VULNERABLE POPULATIONS}

doi:10.1136/sextrans-2013-051184.0890

II Uccella, ${ }^{2} \mathrm{M}$ Cammilli, ${ }^{3} \mathrm{~S}$ Viale, ${ }^{3} \mathrm{C}$ Fazioli, ${ }^{3} \mathrm{R}$ Testa, ${ }^{3} \mathrm{C}$ Mirisola. ${ }^{1}$ National Institute for Health, Migration and Poverty, Roma, Italy; ${ }^{2}$ National Institute for Health, Migration and Poverty, roma, Italy; ${ }^{3}$ National Institute for Health, Migration and Poverty, roma, Italy

Background Despite ongoing prevention and educational efforts, the incidence of new HIV infections in 2011 in Italy is estimated at 3.9 per 100,000 and 21.0 per 100,000 among foreigners. Many new infections, more than half in AIDS stage (sex risk 78.8\%), are caused by persons unaware of their HIV infection.

Methods On February 2012, the NIHMP (National Institute for Health, Migration and Poverty, Rome) started a project aimed at promoting access of vulnerable people to HIV testing and at disseminating knowledge about STIs.

A Rapid HIV-1 Antibody Test (MedMira, Halifax, Nova Scotia) is offered to patients of the infectious diseases unit together with STI counselling involving doctor, nurse, transcultural mediator and psychologist. The test is also proposed to people who had never performed it before. If the test is positive, a confirmatory venous test is required. Multilingual written consent and pre-counselling questionnaire about HIV/STI-related knowledge and stigma and sexual behaviours are provided. After 3-6 months, post-counselling questionnaire is administered.

Results At November 2012, 121 people were enrolled: 72 males (59.5\%), 1 transgender, 103 migrants $(85.1 \%), 9$ homeless people (7.4\%), 4 Roma (3.3\%). 118 people accepted to undergo the test. 61 migrants $(59.8 \%)$ performed it for the first time. The test was offered for screening (54), past STI (22), including two cases of HIV positivity, new STI (21) and STI risk (13). Two AIDS cases were reported (1.6\%). Two tests $(1.69 \%)$ were false positive. 8 tests $(6.7 \%)$ were not defined because of previous positive laboratory HIV test/ negative WB, co-occurrence of hepatitis, syphilis and scabies or aspirin treatment. 85 questionnaires $(70.2 \%)$ were filled in. Couple counselling was conducted in 4 cases.
Conclusions Preliminary data show that rapid testing is accepted and effective as well as laboratory test. The experimental counselling approach for mobile populations involving transcultural mediators will be evaluated.

\section{P3.441 ACCESS TO SERVICES FOR HIV PREVENTION IN MEN IN LONG-DISTANCE DRIVERS GUATEMALA 2012 - 2013}

doi:10.1136/sextrans-2013-051184.0891

'N J Arambu, 'B E Alvarez, 'I Loya-Montiel, 2Z Pinzon, 'B Huaman, ${ }^{3} \mathrm{M}$ Mansur, ${ }^{3} \mathrm{~S}$ Ortega, 3J R Vega, "I Osuna-Ramírez, 'S Morales-Miranda. 'Unidad de VIH, Centro de Estudios en Salud, Universidad del Valle de Guatemala, Guatemala, Guatemala, ${ }^{2}$ Ministerio de salud de Guatemala, Guatemala, Guatemala, 3HIVOS, Guatemala, Guatemala

Background is key to HIV transmission, monitoring trends in groups of men from the general population, especially those whose mobility features and difficult access to sexual health services specialised transit routes is a constant

Methods From November 2012 - January 2013, men were recruited long-haul drivers as part of the Survey of Prevalence of HIV, syphilis and sexual behaviours in key populations of HIV in Guatemala. We used a convenience sample. All participants fulfilled the eligibility criteria and signed an informed consent, a questionnaire was face to face. All participants had been testing for HIV and syphilis. Data were analysed using STATA 11.1

Results 609 men long-distance drivers participated in the study, the median age was 37 years (IOR 31-46). The Guatemalans were 86.54\%, $98 \%$ married or living with a woman and more than half $(56 \%)$ had completed primary education. Only $9.2 \%$ had participated in activities about HIV in the past year, $22.8 \%$ correctly recognised ways of preventing HIV transmission, a $11.35 \%$ underwent an HIV test in the last 12 months, higher than that found in the general population (4.0\%), but lower than in FSW's clients $(23.5 \%)$. Prevalence in HIV were found in $0.50 \%(0.10-1.44)$ and $1.98 \%(1.02-3.43)$ in syphilis

Conclusions The low prevalence of HIV is similar to that found in the general population of Guatemala (0.8\%). Based on the results of this study primarily: the lack of correct knowledge about HIV and the little assistance to get tested for HIV diagnosis in addition with the UNAIDS recommendations in textbooks on population mobility and AIDS interventions are required to establish this special population is a priority under the bridge populations, for their constant mobility.

\section{P3.442 EXPERIENCE OF SCREENING FOR HEPATITIS C IN AN OXFORDSHIRE PRISON}

doi:10.1136/sextrans-2013-051184.0892

\section{S Duncan, J Sherrard. The Churchill Hospital, Oxford, UK}

Since 2004, we have run a fortnightly in-reach sexual health clinic in a medium security male prison which houses 1200 inmates, with a specific wing for men who have committed drugs related offences. In 2007, we reported that the prevalence of Hepatitis C (HCV) in the prisoners we tested was $9 \%$ [1]. Since then, there has been increased awareness of the burden of HCV in prison settings and new recommendations to increase HCV testing [2].

We aimed to review the current prevalence of HCV in local prisoner sexual health screens, and compare this firstly, to our previous 2007 estimate, and secondly, to that in a contemporary male sexual health clinic population.

Methods We performed a retrospective review of all Hepatitis C antibody tests requested for prisoners and male sexual health clinic patients by our service from 1.09.10 - 30.9.11. Samples were identified by laboratory electronic records and supplementary data was acquired by case note review.

Results HCV antibody screens were performed in 118 prisoners, and 716 men attending our general sexual health clinic. The 